\title{
Spontaneous Sequestration of Bisphosphonate Related Oro-Antral Fistula - A Case Report
}

\begin{abstract}
Complications of bisphosphonate therapy have received increasing attention in the field of dentistry as these drugs can potentially cause osteonecrosis of the Maxilla and Mandible (MRONJ). There have been an increasing number of reports in the literature associating osteonecrosis with patients taking bisphosphonates prior to oral surgery (12). In rare cases the necrotic bone segment is entirely separated from the uninvolved bone, which is known as simultaneous sequestrum. The purpose of this case report is to present a case of spontaneous separation of necrotic bone in the maxillary arch without surgical intervention after sinus augmentation and implant placement, in a patient with a history of treatment with oral bisphosphonates. These results and those of others suggested that already osseointegrated dental implants can also cause the osteonecrosis around the implant after bisphosphonates administration. En block sequestration of bone with or without implant might be one of the characteristic s of implantrelated MRONJ
\end{abstract}

\section{Introduction}

The United States is undergoing a rapid increase in its older population due to increased life expectancy and a greater number of people entering their sixth decade of life [1]. This can be attributed to advances in modern medicine and an increase in knowledge regarding public health. However, aging predisposes this population to increased health risks such as cardiovascular disorders, vision problems, compromised immune systems and osteoporosis. Osteoporosis is defined by the World Health Organization (WHO) as a bone mineral density that is 2.5 standard deviations below peak bone mass as measured by Dual Energy X-Ray Absorptiometry (DEXA). This is the most widely used and thoroughly studied bone density measurement technology today [2]. DEXA is a simple,quick and noninvasive procedure which uses a very small dose of ionizing radiations to measure bone loss. Osteoporosis has been treated with various modalities, which include exercise, increasing protein, calcium, and vitamin D intake, estrogen therapy, Parathyroid Hormone (PTH) and antiresorptive medications such as bisphosphonates [3].

Bisphosphonates are being prescribed more often for patients with osteoporosis to prevent bone fracture. According to the national osteoporosis foundation data released in 2014, 54 million U.S adults age 50 years and older are affected by osteoporosis and low bone mass. As estimated, 10.2 million adults have osteoporosis and 43.4 million have osteopenia (low bone density) which is a precursor condition for osteoporosis [4]. The mechanism of action of bisphosphonates involves complex interactions within the bone. This class of medication causes decreased osteoclastic activity and eventually apoptosis of these cells. New osteoclasts are not formed, therefore reduced bone resorption and increased bone density may reduce the risk of fractures in these

\section{Journal of Oral Biology}

\author{
Muchhala S, Rodríguez-Fernández $\mathbf{M}^{*}$, Barsoum A, \\ Patel V, Fernández-Guallart I and Grasso G \\ Arthur Ashman Department of Periodontology and Implant \\ Dentistry, New York University, New York \\ *Address for Correspondence \\ Rodríguez-Fernández M, Department of Periodontology and Implant \\ Dentistry, New York University, Clinic 5W, 345 E 24th St, NY 10010, \\ New York, Tel: +1-347 27929 58; E-mail: ycy233@nyu.edu \\ Submission: 05-February-2020 \\ Accepted: 31-March-2020 \\ Published: 03-April-2020 \\ Copyright: @ 2020 Muchhala S, et al. This is an open access article \\ distributed under the Creative Commons Attribution License, which \\ permits unrestricted use, distribution, and reproduction in any medium, \\ provided the original work is properly cited.
}

patients. In addition to osteoporosis, bisphosphonates are indicated for treatment of metastatic cancer [5], multiple myeloma [6], Paget's disease [7], or hypercalcemia associated with malignancy [8]. More recently, bisphosphonates have been used to reduce fracture rates in children with Osteogenesis Imperfecta [9].

Although bisphosphonates are beneficial in many ways, they pose a risk to patients taking these medications. Complications of bisphosphonate therapy have received increasing attention in the field of dentistry as these drugs can potentially cause osteonecrosis of the maxilla and mandible [10]. The term Medication- Related Osteonecrosis of the Jaw (MRONJ) was given to this condition by the American Association of Oral Maxillofacial Surgeons [11]. Osteonecrosis of the Jaw is a serious condition that is characterized by a non-healing and often painful exposure of sequestrating bone. The MRONJ lesion is commonly located in areas with dense bone hence MRONJ is more probable in the mandible than the maxilla $(2: 1)$. There have been an increasing number of reports in the literature associating osteonecrosis with patients taking bisphosphonates prior to oral surgery [12].

Depending on the risk and severity of MRONJ, different treatment modalities are recommended including pain medication, antibiotics, antibacterial mouth rinses, surgical debridement and resection of the exposed site [11]. However, in rare cases the necrotic bone segment is entirely separated from the uninvolved bone, which is known as simultaneous sequestrum. To the best of authors knowledge few articles have reported on treatment of simultaneous sequestrum in the maxilla [13].

The purpose of this case report is to present a case of spontaneous separation of necrotic bone in the maxillary arch without surgical intervention after sinus augmentation and implant placement, in a patient with a history of treatment with oral bisphosphonates.

\section{Materials and Methods}

The clinical data in this case report was extracted from the anonymous Implant Database (ID) from the routine treatment of patients at the Ashman Department of Periodontology and Implant Dentistry at the New York University College of Dentistry Kriser 
Citation: Muchhala S, Rodríguez-Fernández M, Barsoum A, Patel V, Fernández-Guallart I, et al. Spontaneous Sequestration of Bisphosphonate Related Oro-Antral Fistula - A Case Report. J Oral Biol. 2020; 7(1): 5.

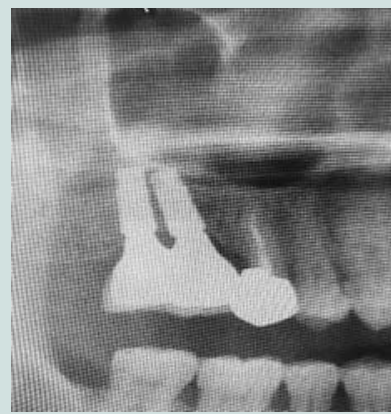

Figure 1: Showing Implant with sinus augmentation and final restoration

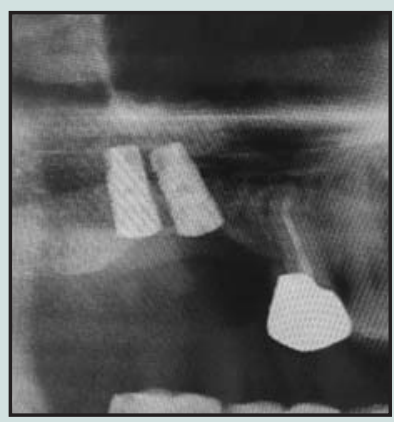

Figure 2: Periimplantitis on \#2,3 implants.

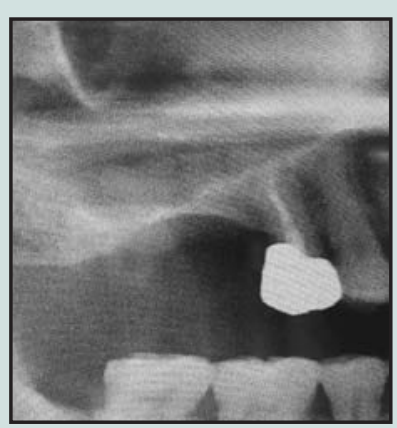

Figure 3: After Implants were explanted due to pain and periimplantitis

Dental Center. The office of Quality Assurance at the New York University College of Dentistry certified the ID. This case report is in compliance with the Health Insurance Portability and Accountability Act (HIPPA) requirements and approved by the University Committee on the Activities Involving Human Subjects (UCAIHS).

\section{Case Report}

In August 2004, a 50-year-old Asian female patient, with a 10year history of taking Ibandronate sodium (Boniva150mg/4weeks orally), for treatment of osteoporosis, presented to the Ashman Department of Periodontology and Implant Dentistry of New York University College of Dentistry. Her chief complaint was replacement of teeth with fixed prosthesis on missing maxillary right, first and second molars and second premolar. Intraoral evaluation showed a healthy periodontium. Periapical radiographs made using paralleling cone technique showed adequate restorative space but lack of apico- coronal bone height for implant placement. A cone beam computer tomography was taken to confirm the same.

After temporary cessation of the antiresorptive medication for 3 months before the scheduled surgery, a sinus augmentation procedure was done via lateral window approach. The Schneiderian membrane was assessed visually and then was elevated without any perforation noted. The initial osteotomy was made considering optimal angulation and position for the planned final implants, and the graft material (Geistlich Bioss large particle) was placed in the contained space. Two parallel wall implants (Osseotite 3i Zimmer) were placed in the osteotomy in site \#2 and \#3. Chromic gut (4-0) sutures (Henry Schein, Melville, N.Y, U.S.A) were used to achieve tension-free primary closure. The patient was put on pre - operative ( $2 \mathrm{~g}$ amoxicillin $1 \mathrm{~h}$ prior to procedure) and post-operative antibiotics (500mg amoxicillin/8h/7days). Regular follow-ups showed uneventful healing. Six months later the final restoration was delivered. Patient maintained good oral hygiene and regular follow-ups for one year (Figure 1).

In 2013 the patient presented to the clinic after 7 years of missed follow-ups and was diagnosed with peri-implantitis in site \#2 (Figure 2). An updated medical history revealed that the patient was now

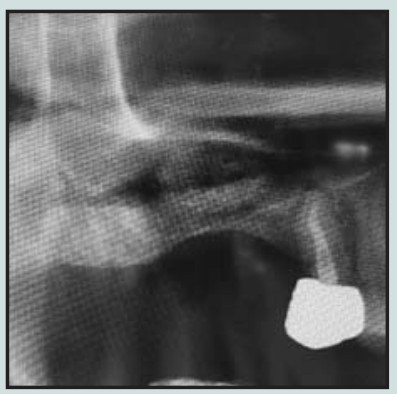

Figure 4: X-ray showing bony segment separated from surrounding bone.

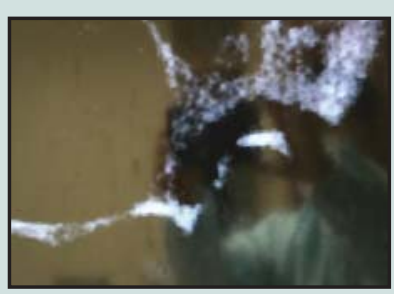

Figure 5: CT scan showing sequestrated bony segment separated from the surrounding bone.

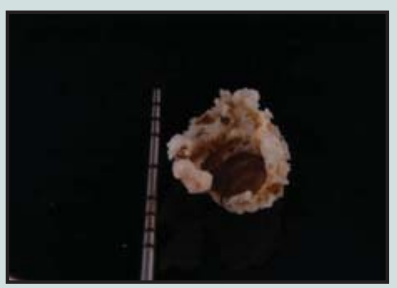

Figure 6: Sequestrated osteonecrotic bone. 


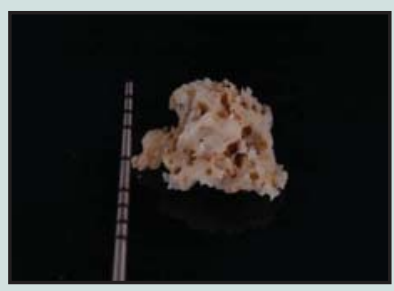

Figure 7: Sequestrated osteonecrotic bone.

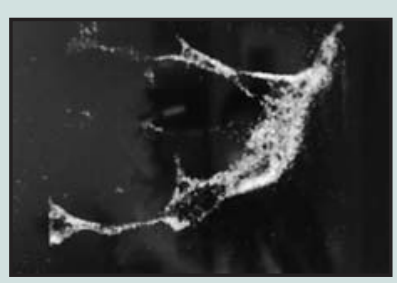

Figure 8: CT scan showing healed bone after the sequestrated bone was removed.

taking Atelvia (Risedronate sodium $35 \mathrm{mg} /$ week orally). The fixed restoration was removed and the implant in site \#2 was explanted due to peri-implantitis and pain. Three years later, in 2016,the implant on site \#3 had to be removed as well due to the same reason(Figure 3).

Two months after removal of the implant \#3, the patient returned with edematous tissue in the maxillary first molar region. The tissue was excised, drained and irrigated and patient was put on amoxicillin (500 mg/7 days). The same procedure was repeated in the next few visits, but the site did not show improvement. Evidence of bony sequestrum was seen four months after the explantation of the implants (Figures 4 and 5). Upon administration of local anesthesia (Lidocaine 1:100,000) the sequestrated bony segment was removed and the patient healed uneventfully (Figure 6-9).

Six months later, two implants $3.3 \times 10 \mathrm{~mm}$ (EBI, Kyungsan, Republic of Korea) were placed in site \#2 and \#3. Osseointegration was achieved. 3-year follow-up showed successful results(figure 10). The patient was put in intervals of 6 months.

\section{Discussion}

Bisphosphonates are widely used to treat systemic metabolic bone diseases. However, their use predisposes a patient to the potential complication of Medication-Induced Osteonecrosis of the Jaw (MRONJ). This complication must be considered in the various disciplines in the dental field, including implant dentistry, oral surgery, periodontics and endodontics.

In MRONJ, alveolar bone becomes exposed for more than 8 weeks spontaneously, or after invasive surgical procedures such as implant placement, tooth extraction, periodontal surgery or apicoectomy. The osteonecrosis originates in the alveolar bone and extends into the basal bone. The diagnosis is based on clinical findings. However, there are radiographic, histological, and microbiological findings associated with MRONJ, which are not pathognomonic for the condition. Radiographic signs include sclerosis or loss of the lamina dura, and/ or widening of the periodontal ligament space. Microscopically the appearance of necrotic bone with some bacterial colonization has been noted [9]. The 3 most common species associated with secondary infection in exposed bone due to bisphosphonates are actinomices, eikenella, and moraxell [12]. Intravenous (IV) bisphosphonates such as Aredia (pamidronate) or Zometa (zoledronate) cause osteonecrosis of the jaws from 6 to 16 months post drug treatment [13]. Reported rates of MRONJ with IV bisphosphonates range from $0.8 \%$ to $12 \%$ [14]. In contrast, osteonecrosis caused by oral bisphosphonates may occur 3 years or more after administration due to their lower absorption rate [15]. Reported rates of spontaneous MRONJ with oral drugs such as Ibandronate sodium, risedronate and alendronate were 0.01 to $0.04 \%$ and increased to 0.09 to $0.34 \%$ after dental extractions [16]. A study which analyzed 119 patients on IV bisphosphonate therapy in 2005 found that nearly $38 \%$ of bone exposure was associated with extractions and small percentage (3.4\%) was associated with implant placement [13]. On the other hand, in a study published by Jeffcoat in 2006, not a single case of MRONJ was reported following the placement of 210 implants in 50 patients taking the oral form bisphosphonates [17]. Merck Inc. has received reports of 170 cases of MRONJ associated with oral

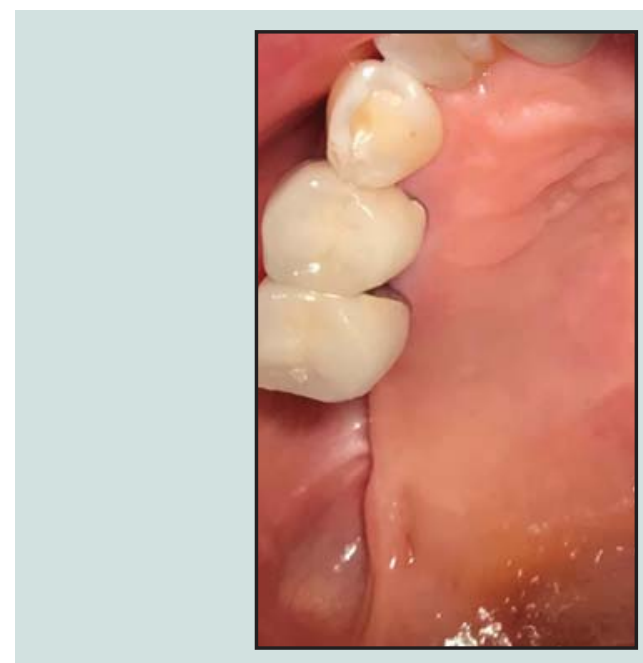

Figure 9: Intraoral picture of healed ridge after removal of implants and bony segment.

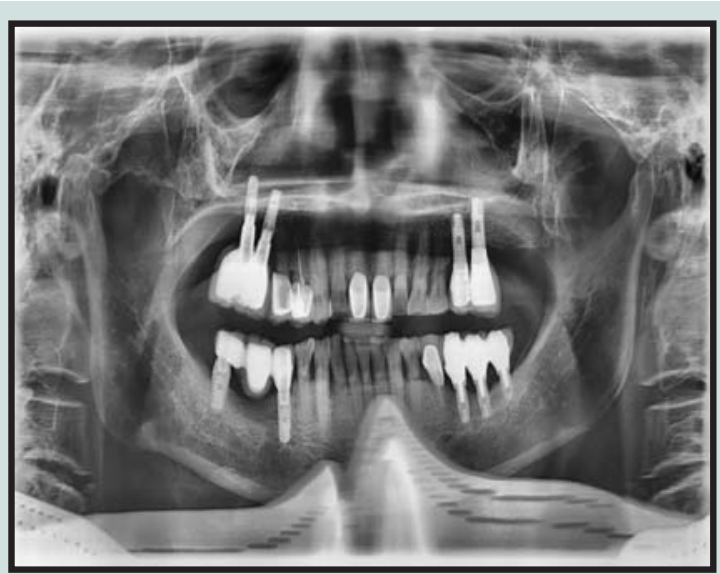

Figure 10: Three years post-operative panoramic x-ray. 
Citation: Muchhala S, Rodríguez-Fernández M, Barsoum A, Patel V, Fernández-Guallart I, et al. Spontaneous Sequestration of Bisphosphonate Related Oro-Antral Fistula - A Case Report. J Oral Biol. 2020; 7(1): 5.

alendronate therapy out of approximately 20 million patient-years, or 0.7 reports of MRONJ per 100,000 patient-years of exposure. With oral risedronate, 1 to 1.2 cases per 10,000 patient-years were reported by Procter \& Gamble. Current American Association of Oral and Maxillofacial Surgeons guidelines recommend a drug holiday from 3 to 6 months before dental implant placement in patients with a history of oral bisphosphonate used for greater than 3 years.

It is estimated that $75 \%$ of MRONJ is caused by uncontrolled dental inflammatory disease and dental trauma. Therefore, avoiding invasive dental surgery is highly recommended after drug therapy has been initiated. The absorption of oral bisphosphonates is much slower than the IV form, so the risk of MRONJ usually does not manifest itself until after 3 years of continuous administration [15]. Dental treatment, including implants is not contraindicated within this 3 year window [18]. However, physicians may not be inclined to take patients off of bisphosphonate therapy due to increased morbidity and mortality rates associated with osteoporotic fractures $[19,20]$. Patients who cannot alter their drug regimen and are in need of emergency care should be treated with informed consent which describes the potential to develop osteonecrosis of the jaws. Marx has advocated a method of assessing risk for patients using oral bisphosphonates [9]. He recommends that the patient receive a blood test for serum CTX (C-telopeptides of type I collagen) which is a bone turnover marker. This test can identify changes in bone remodeling/renewal within 2 days to 2 weeks of onset of drug use. This test measures the relative rate of bone renewal and bone resorption. Values greater than $150 \mathrm{mg} / \mathrm{ml}$ correlate to low risk for developing osteonecrosis. Values between 100 and $150 \mathrm{mg} / \mathrm{ml}$ correlate to a moderate risk, while values below $100 \mathrm{mg} / \mathrm{ml}$ identify high risk patients. However, the accuracy of this test has been questioned and there have been no long-term studies to measure its validity, specificity and accuracy.

Treatment should be rendered in cases where bone has been exposed for more than 8 weeks. Management of the patient depends on the clinical severity of the exposed bone and presentation of local/systemic infection. Intravenous bisphosphonate-induced osteonecrosis usually presents as more clinically extensive and less responsive to treatment than oral bisphosphonate-induced osteonecrosis [13]. The following four categories of treatment are proposed by the American Association of Oral Maxillofacial Surgery Position Paper on treatment of MRONJ. The preliminary one includes patients at risk of developing MRONJ due to the fact that they have been treated with oral or intravenous bisphosphonates, and only requires prevention such as avoidance of traumatic surgery. The first true category (stage 1), when asymptomatic necrotic bone is exposed around implants, suggests a treatment of daily rinse with $0.12 \%$ chlorohexidine. The second category (stage 2 ) is seen in patients with exposed bone associated with infection and pain. In this case, antibiotics should be considered in addition to daily rinses with $0.12 \%$ chlorohexidine and proper analgesics. The primary antibiotic that is suggested is penicillin (Pen VK $500 \mathrm{mg}$ ) prescribed four times daily due to its significant effect on the three main species of bacteria present secondary to osteonecrosis. Other antibiotics include levofloxacin (Levaquin 500mg once daily) or azithromycin (Zythromax250 mg once daily). Antibiotic regimens are given for 14 days. In cases of refractory pain, Flagyl (Metronidazole500 mg) can be prescribed three times a day. Incision and drainage of the area should be completed when fluctuant swelling is present. The third category of patients (stage 3 ) presents with additional clinical signs such as mobility of the exposed bone, extra-oral fistula, pathologic fracture or osteolysis extending to the inferior border, and its treatment includes surgical debridement and resection for longer term palliation of infection and pain after drug therapy (antibiotic and painkillers, as well as antibacterial mouth rinse) has been initiated [16].

Good communication with health professionals is essential on deciding the most appropriate timing of dental treatment. If possible, IV bisphosphonate therapy should be withheld for roughly 2-3 months while attending to the patient's dental needs. For patients not requiring invasive dental therapy, IV bisphosphonate therapy does not need to be delayed. If a patient is currently on bisphosphonate therapy, the aim of dental treatment is to maintain optimal oral health and to avoid invasive dental procedures. For example, a tooth that is deemed unrestorable due to caries should have endodontic therapy completed and the crown amputated as opposed to it being extracted. If a tooth must be extracted, penicillin (Pen VK $500 \mathrm{mg}$ prescribed four times daily) or azithromycin (Zythromax $250 \mathrm{mg}$ once daily) should be prescribed for 7 days. Periodontal disease should be treated non-surgically with supragingival scaling and irrigation with $0.12 \%$ chlorohexidine rinse [18].

Several factors could have played a role in the development of MRONJ in our patient. Although Bisphosphonates tend to accumulate in sites of active bone remodeling like the jaws, the surgical trauma to the alveolar bone during implant surgery could have further stimulated the postoperative accumulation of the drug in the implanted site. However, our patient had stopped oral bisphosphonate therapy for 3 months prior to sinus grafting procedure and dental implants placement and still developed MRONJ after 7 years. The terminal (slowest) elimination half-life was estimated to be about 10 years, consistent with the overall estimated half-life of skeletal turnover. This could be another reason for MRONJ development in our patient. MRONJ is more often localized in the mandible than in the maxilla (2:1 ratio). A compromised blood supply to the maxilla and mandible, along with altered bone remodeling after exposure to long-term Bisphosphonate therapy, has been proposed as the theory behind the pathogenesis of osteonecrosis [21,22]. In the present case, the fact that the episode occurred in the maxilla could be related to the avascular nature of the sinus cavity, specially after being grafted with xenograft. In this case, the exposed bone could have been classified as a stage 3 due to its mobility, and was treated with removal and debridement of the area, antibiotics, painkillers and mouthwash. After its complete healing and monitoring for 6 more months, the area was considered suitable for a new minimally invasive implant treatment.

\section{Conclusion}

Few reports describe side effects of bisphosphonate usage, particularly such as MRONJ in the maxilla. This case addresses this lack. Presentation of MRONJ is currently more commonly seen in patients taking the intravenous forms of the drug; however, prescriptions for oral bisphosphonates continue to rise, and there is fear that the long-term cumulative effects of these drugs may see MRONJ occurring in patients at a rate equal to that seen in patients 
Citation: Muchhala S, Rodríguez-Fernández M, Barsoum A, Patel V, Fernández-Guallart I, et al. Spontaneous Sequestration of Bisphosphonate Related Oro-Antral Fistula - A Case Report. J Oral Biol. 2020; 7(1): 5.

undertaking intravenous therapy. Good communication with health professionals is essential on deciding the most appropriate timing of dental treatment. Many of the precautions taken in order to reduce the risk of MRONJ with IV bisphosphonate therapy should also be followed in patients taking oral bisphosphonates. In conclusion, these results and those of others suggested that already osseointegrated dental implants can also cause the osteonecrosis around the implant after bisphosphonates administration. En block sequestration of bone with or without implant might be one of the characteristics of implant-related MRONJ.

\section{References}

1. Rice D, Fineman N (2004) Economic implications of increased longevity in the United States. Annu Rev Public Health 25: 457-473.

2. Gilsanz V (1998) Bone density in children: A review of the available technique and indications. Eur J Radiol 26: 117-182.

3. Zhang J, Saag GK, Curtis JR (2011) Curtis, Long term concerns of antiresorptive therapy. Rheum Dis Clin North Am 37: 387-400.

4. Wright N, Looker A, Saag K, Curtis J, Delzell E, et al. (2014) Recent prevelance of osteoporosis and low bone mass in the United States based on bone mineral density at femoral, neck or Lumbar spine. Journal of Bone and Mineral Research 29: 2520-2526.

5. Hortobagyi G, Theriault R, Porter L, Blayney D, Lipton A, et al. (1996) Efficacy of pamidronate in reducing skeletal complications in patients with breast cancer and lytic cone metastasis. New England Journal of Medicine 335: 1785-1791.

6. Berenson J, Lichtenstein A, Porter L, Dimopoulos M, Bordoni R, et al. (1996) Efficacy of Pamidronate in Reducing Skeletal Events in Patients with Advanced Multiple Myeloma. New England Journal of Medicine 334: 448493.

7. Chapurlat R, Meunier P (1998) Bisphosphonates and bone remodeling effectiveness in Paget's disease, fibrous dysplasia, and osteoporosis. Rev Chir Orthop Reparatrice Appar Mot 84: 743-751.

8. Mayor P (2002) The use of Zeledronic acid, a novel, highly potent bisphosphonate, for the treatment of hypercalcemia of malignancy. Oncologist 7: 481-491

9. Glorieux F, Bishop N, Plotkin H, Chabot G, Lanoue G, et al. (1998) Cyclic administration of pamidronate in children with severe osteogenesis imperfecta. N Engl J Med 339: 947-952.

10. Marx. RE, Cillio JI jr, Ullao JJ (2007) Oral Bisphosphonate-Induced Osteonecrosis: risk factors, prediction of risk using serum CTX testing, prevention and treatment. J Oral Maxillofac Surg 65: 2397-2410.

11. Salvatore R, Thomas BD, Jhon F, Reginald G, Tara A, et al. (2014) American Association of Oral and Maxillofacial Surgeons Position Paper on MedicationRelated Osteonecrosis of the jaw--2014 Update 72: 1938-1956.

12. Bedogni A, Totola A, Bettini G, Saia G, Nocini (2010) Oral bisphosphonateassociated osteonecrosis of the jaw after implant surgery a case report and literature review. J Oral Maxillofac Surg 68: 1662-1666.

13. Mohammad. H, Bainton R, Srinivasan D (2011) Non surgical management of stage 3 bisphosphonate related oroantral fistula. Br J Oral Maxillofac Surg 49: e16-e17.

14. Hoff A, Toth B, Hortobagyi G, Gagel R, Luna M (2005) Retrospective chart review to collect information on the frequency of osteonecrosis in patients treated with IV bisphosponate therapy. Houston, TX: U. of Texas, M.D. Anderson Cancer Center 2005 1: 1-5.

15. Yarom N, Yahalom R, Shoshani Y, Hamed W, Regev E, et al. (2007) Osteonecrosis of the jaw induced by orally administered bisphosphonates: incidence, clinical features, predisposing factors and treatment outcome. Osteoporosis International 18: 1363-1370.

16. (2006) American Association of Oral and Maxillofacial Surgeons Position Paper on Bisphosphonate-Related Osteonecrosis of the Jaws. J Oral Maxillofac Surg 65: 369-376.

17. Jeffcoat MK (2006) Safety of oral bisphosphonates: controlled studies on alveolar bone. Int J Oral Maxillofac Implants 21: 349-353.

18. Grant BT, Amenedo C, Freeman K, Krant RA (2008) Outcomes of Placing Dental Implants in Patients Taking Oral Bisphosphonates: A Review of115 Cases. J Oral Maxillofac Surg 66: 223-230.

19. Rabenda V, Mertens R, Fabri V, Vanoverloop J, Sumkay F, et al. (2007) Adherence to bisphosphonates therapy and hip fracture risk in osteoporotic women. Osteoporosis International 26: 315-319.

20. Greenspan S, Harris S, Bone H, Miller P, Orwoll E, et al. (2001) Bisphosphonates: Safety and Efficacy in the Treatment and Prevention of Osteoporosis. American Family Physician 63: 1913-1914.

21. Gupta S, Gupta H, Mandhyan D, Srivastava S (2013) Bisphophonates related osteonecrosis of the jaw. National journal of maxillofacial surgery 4: 151

22. Bedogni A, Bettini G, Totola A, Saia G, Nocini PF (2010) Oral Bisphosphonateassociated osteonecrosis of the jaw after implant surgery: A case report and literature review. Journal of Oral and Maxillofacial Surgery 68: 1662-1666. 